\title{
The rate of manual peripheral blood smear reviews in outpatients
}

\author{
Paul Froom*, Rosa Havis and Mira Barak \\ Central Laboratory, Haifa and the Western Galilee, \\ Clalit Health Services, Nesher, Israel
}

\begin{abstract}
Background: The International Consensus Group for Hematology Review recommends manual blood smears for new findings from automated complete blood cell (CBC) tests. However, in outpatients, the rate of peripheral manual blood smear reviews is uncertain, as is the effect of historical results on this rate.

Methods: In a prospective cohort study of 687,955 members of a health maintenance organization, we extracted 39,759 consecutive automated CBC test results and determined the rate of manual peripheral smear reviews that were indicated using different triggering criteria, and historical results.

Results: Individual triggering values increased from $10.7 \%$ to $19.5 \%$ when comparing our criteria to those of the consensus criteria. The proportion of CBC test results with at least one triggering value was $7.2 \%$ using our criteria, compared to $13.9 \%$ with the inclusion of a mean corpuscular volume $<75$ and a plus one immature granulocyte flag according to the consensus committee. Availability of historical data decreased our peripheral smear review rate to $2.6 \%$. Conclusions: The peripheral smear review rate in outpatients using the consensus criteria was higher than with our less stringent criteria. If historical data are available and no clinical utility is demonstrated for the additional consensus criteria, an acceptable outpatient review rate might be as low as $2.6 \%$. Clin Chem Lab Med 2009;47:1401-5.
\end{abstract}

Keywords: manual; outpatient; peripheral; smear review; triggering values.

\section{Introduction}

Automated laboratory hematology analyzers provide cell counts, flags, cell plots (instrumental morphology) and distributional histograms with greater precision and accuracy than that provided by manual determinations $(1,2)$. However, for pathological conditions such as acute leukemia, a manual peripheral

\footnotetext{
*Corresponding author: Professor P. Froom, MD, Head of the Hematology Laboratory, Central Laboratory of Haifa and Western Galilee, Clalit Health Services, Nesher, Israel Phone: +972-4-6394470, Fax: +972-4-6243302

E-mail: paulfr@clalit.org.i

Received May 17, 2009; accepted July 23, 2009; previously published online September 25, 2009
}

blood smear review is required to make the presumptive diagnosis. Also, there are other findings usually not reliably identified by the various automated analyzers that might have clinical utility. These other findings include the presence of platelet clumps, basophilic stippling, hyper-segmented neutrophils, red-cell fragments, giant platelets, and Howell-Jolly bodies (3).

Determining acceptable rates for performing manual blood smears is important to ensure the quality of the reported complete blood count (CBC) results, but reported rates are highly variable. In a study of 263 institutions serving both inpatients and outpatients, the 10th-90th percentile smear review rates were $9.9 \%-50 \%$ (4). The variability was probably due to the patient mix and to differences in criteria for triggering manual reviews. In fact, the 10\%-90\% triggering percentiles ranged from 15 to $20 \times 10^{9}$ cells/L for a high white blood cell count (WBC), $1.5-3.0 \times 10^{9}$ cells/L for a low WBC, $30-100 \times 10^{9} / \mathrm{L}$ for low platelets counts, $600-1000 \times 10^{9} / \mathrm{L}$ for high platelets counts, $65-100 \mathrm{~g} / \mathrm{L}$ for low hemoglobin values, 100-120 fL for a high mean corpuscular volume (MCV), and from 65 to $79 \mathrm{fL}$ for a low MCV (4). Consensus criteria by the International Consensus Group for Hematology Review (5) fall within the above ranges, but they added a caveat that nearly all criteria apply only to the first time that the triggering value is observed. The impact of this recommendation on manual blood smear review rates is unclear.

In the following study, we report the effect of various criteria and the availability of historical data on manual peripheral blood smear review rates in 39,759 consecutive automated CBC tests performed during December 2008 in a well-defined outpatient population.

\section{Materials and methods}

\section{Design overview}

We obtained the age and gender of all active members of the Clalit Health Services-Haifa and Western Galilee during December 2008. The number and proportions of CBC tests done during 2008 were calculated for each age group. We identified all cases of acute leukemia, new onset chronic myeloid leukemia, autoimmune hemolytic anemia, and $\beta$ thalassemia minor identified during the entire year, and reviewed all $\mathrm{CBC}$ tests performed during the 3 months prior to the diagnosis.

During the month of December, $\mathrm{CBC}$ test results, peripheral smear reviews, age and gender for all samples were extracted from our database. CBC tests sent to the laboratory were performed using an Advia120 or Advia2120 analyzer (Siemens Healthcare Diagnostics, Deerfield, IL, USA). The quality control program included daily harmonization pro- 
cedures, as well as internal and external (UK NEQAS) quality control assessments. Our mean total errors were as follows: WBC: $5.7 \%$, red blood cell count (RBC): $2.2 \%$, hemoglobin: 2.8\%, MCV: $2.7 \%$, and platelets: $7.4 \%$. Differential cell counts were not included in the external quality control programs, but imprecision was: neutrophils: $5 \%$, lymphocytes: $3.5 \%$, monocytes: $17.8 \%$, eosinophils: $22.2 \%$, and basophils: $32.5 \%$. Tests sent to the laboratory were analyzed within $8 \mathrm{~h}$ following phlebotomy.

Validation procedures also included repeating the analysis on a different analyzer when a hemoglobin value was $<70 \mathrm{~g} / \mathrm{L}$ in adults or $<90 \mathrm{~g} / \mathrm{L}$ in children ( $<$ age 13 years old), irregardless of previous testing, repeating a platelet count $<40$ or $>1000 \times 10^{9} / \mathrm{L}$, and a first time WBC of $>30 \times 10^{9}$ cells/L or $<1.5 \times 10^{9}$ cells/L. Patients without a history of anemia had a reticulocyte count performed if the hemoglobin value was $<100 \mathrm{~g} / \mathrm{L}$ and the MCV was $80 \mathrm{fL}$ or greater (6). A direct antiglobulin test was performed if the uncorrected reticulocyte count was $2 \%$ or more of the RBCs (6). Hemoglobin electrophoresis was done if requested by the physician or if the patient had an MCV $<75 \mathrm{fL}$, a $\mathrm{RBC}$ value $\geq 4.5$ cells $\times 10^{12} / \mathrm{L}$, and no previous results.

Historical data were available for a 4-year period. Smears were performed if there was a new triggering value not present during the previous two years. Exclusion patients included 25 individuals aged 100 or more (because of small numbers), and test results with one or more missing values.

\section{Analysis}

The proportion of triggering values according to our criteria was compared to those of the consensus committee. We calculated the number of individual triggering values for each criterion (Table 1).

Next, we calculated the proportion of $\mathrm{CBC}$ tests with at least one criterion. We considered first our criteria, and then added the indications recommended by the international consensus committee. Finally, we determined the effect of having historical data available on the peripheral smear rate (at least one of the criteria not present in the preceding 2 years). The proportion of smears performed included all peripheral slide reviews (both differentials and smear scans). The $\chi^{2}$-test was used to calculate the $p$-value for categorical data.

\section{Ethical considerations}

The data for this study are part of our quality management system. Use of the data is in accordance with the ethical standards established by Clalit Health Services.

\section{Results}

The active members of our health maintenance organization included 353,780 females and 334,175 males, aged $<1$ to over 100 years old. The age distribution was as follows: < 15 years, $24.3 \% ; 15-34$ years, $29.7 \%$; $35-54$ years, $22.2 \%$; $55-74$ years, $16.8 \%$; and 75 years old or more, $6.9 \%$. There were 0.84 CBCs done per female and 0.55 per male $(p=0.0011)$ during 2008. The proportion increased with age and was higher in females than in males until age 74 , as would be expected from known gender differences in the use of health care services (7). Sixty-one percent of patients with a CBC request in December were female, and their mean age was $52 \pm 24$ years. We found only minor seasonal variations in the triggering rate for manual smears, warranting the extrapolation of December's results to the rest of the year (results not shown). There were 842 exclusions because of one or more missing values, and after adding the 25 exclusions because of age ( $>99$ years old), there were 39,759 results from a total of 40,626 CBC tests that were performed (97.9\%). This corresponds to $\sim 1800$ CBC tests per day performed in the hematology laboratory. The laboratory also performs $\sim 70$ reticulocyte counts, and 70 hemoglobin electrophoresis tests per day during our 5-day work week.

Table 1 Individual triggering values, comparing our criteria to those of the consensus committee in 39,759 complete blood cell test results.

\begin{tabular}{|c|c|c|c|c|}
\hline Test, units & Consensus & Our criteria & Consensus, $\mathrm{n}$ & Our criteria, $\mathrm{n}$ \\
\hline WBC, $\times 10^{9}$ cells $/ \mathrm{L}$ & $>30$ & $>20$ & 80 & 188 \\
\hline WBC, $\times 10^{9}$ cells $/ \mathrm{L}$ & $<4.0$ & $<3.8$ & 807 & 588 \\
\hline Platelet count, $\times 10^{9} / \mathrm{L}$ & $<100$ & $<100$ & 364 & 364 \\
\hline Platelet count, $\times 10^{9} / \mathrm{L}$ & $>1000$ & $>1000$ & 15 & 15 \\
\hline Hemoglobin, g/L & $<70$ & $<70$ & 28 & 28 \\
\hline $\mathrm{MCV}, \mathrm{fL}$ & $<75$ & - & 2059 & - \\
\hline$M C V, f L$ & $>105$ & $\geq 100$ & 130 & 545 \\
\hline RDW, \% & $>22$ & - & 56 & - \\
\hline Neutrophils, $\times 10^{9}$ cells $/ \mathrm{L}$ & $<1$ & $<1$ & 148 & 148 \\
\hline Lymphocytes, $\times 10^{9}$ cells $/ \mathrm{L}$ & $>5$ & $>5$ & $384^{\mathrm{b}}$ & 881 \\
\hline$<12$ years old, $\times 10^{9}$ cells $/ \mathrm{L}$ & $>7$ & $>5$ & $157^{\mathrm{b}}$ & - \\
\hline Monocytes, $\times 10^{9}$ cells $/ \mathrm{L}$ & 1.5 & $>12 \%$ & 33 & 280 \\
\hline$<12$ years old, $\times 10^{9}$ cells $/ \mathrm{L}$ & 3 & - & 0 & - \\
\hline Eosinophils, $\times 10^{9}$ cells $/ \mathrm{L}$ & 2 & - & 41 & - \\
\hline Basophils, $\times 10^{9}$ cells $/ \mathrm{L}$ & 0.5 & - & 41 & - \\
\hline Nucleated RBCs, Plus & + & - & 466 & - \\
\hline Immature, Plus & + & +++ & 1615 & 163 \\
\hline Atypical - flaga ${ }^{\mathrm{a}}$, Plus & + & LUC $^{a} \geq 5 \%$ & 948 & 668 \\
\hline Blast flag, Plus & + & + & 363 & 363 \\
\hline Total, \% & - & - & 7735 (19.5\%) & $4231(10.6 \%)$ \\
\hline
\end{tabular}

${ }^{a}$ Atypical - flag (LUC - large unstained cells, $4 \%$ corresponds roughly to the atypical lymphocyte flag of one plus). ${ }^{b}$ For the consensus criteria, the numbers of adults and children need to be considered together to compare to our criteria (the same for children and adults). WBC, white blood cells; RBC, red blood cells; RDW, red cell distribution width. 
The total number of individual criteria influencing the smear review rate was $10.6 \%$ using our criteria, compared to $19.5 \%$ using the criteria of the consensus committee (Table 1). Our criteria were the same as those of the consensus committee for platelet counts, hemoglobin values, for low absolute neutrophil counts, and for presence of blast flags. We used stricter criteria leading to less peripheral blood smear reviews for a low total WBC, atypical lymphocytes [flag or percent of large unstained cells (LUCs)], and immature granulocyte flags. We did not do smear reviews for microcytic anemia, or the presence of nucleated RBCs. Also, we did not perform smears for elevated absolute esoinophil or basophil counts.

However, our criteria were more sensitive for the absolute lymphocyte counts. We used the same criteria for adults and children, whereas, the consensus committee used a higher cut-off for absolute counts in children. Also, we used a lower cut-off for a high $\mathrm{MCV}$, and the proportion of monocytes that increased the peripheral blood smear review rate.

$\mathrm{CBC}$ tests with one or more criteria increased from $7.2 \%$ using our criteria to $13.9 \%$ using the recommendations of the consensus committee (Table 2), predominantly due to review of smears with an MCV of $<75 \mathrm{fL}$, or those with a plus one rather than plus three immature granulocyte flag. Use of historical data decreased the smear review rate using our criteria from $7.2 \%$ to $2.6 \%$.

There were $672(1.7 \%)$ additional smear reviews performed without being flagged by criteria alerts. These were the result of direct requests by the physician or laboratory technicians. Requests by physicians rarely included a suspected diagnosis and our data system does not provide a clinical history. Physician requests included a single request to look for acanthocytes (not found), Sezary cells (found in 2 of 20 patients with Mycosis Fungoides), and red cell fragments found in 1 of 11 requests. No significant findings were found in remaining smear reviews.

The highest prevalence of at least one triggering value was found in those individuals under the age of
10 years. The major reason for the increased prevalence was the presence of an increase in the absolute lymphocyte count, the presence of increased LUC proportions, flags for blasts and flags for immature granulocytes (Figure 1).

During 2008, the detection of 19 of 24 acute leukemia patients was triggered by the criteria of the basic parameters alone (hemoglobin $<70 \mathrm{~g} / \mathrm{L}$, WBC $<3.8 \times 10^{9} \mathrm{cells} / \mathrm{L}$ WBC $\geq 20 \times 10^{9} \mathrm{cells} / \mathrm{L}$ or platelets $\left.<100 \times 10^{9} / \mathrm{L}\right)$. None of these patients were under 20 years of age. A blast flag was found in 22 cases, LUC $>5 \%$ in 20 cases and a three plus immature granulocyte flag in 10 of the 24 patients. Five patients without any of the basic criteria had both increased LUC proportions and blast flags. The morphology of 22 of the 24 acute leukemia patients appeared to be nonlymphocytic, and the two cases of lymphocytic morphology were confirmed by flow cytometry. There was one patient with acute leukemia who had a manual peripheral blood smear review triggered because of a finding of $10 \%$ LUC three weeks before the diagnosis was made from a repeat $\mathrm{CBC}$ test. There were no blasts or atypical lymphocytes identified on manual examination. There were three new cases of CML diagnosed by peripheral smear review. Even though there are findings suggestive of other myeloproliferative diseases, myelodysplastic syndrome and chronic lymphocytic leukemia, we did not try to determine the incidence of these diseases because diagnosis and treatment are not based on such findings. Reflex testing identified 32 new cases of autoimmune hemolytic anemia and 135 new cases of $\beta$-thalassemia minor $(67.8 \%$ of those tested).

\section{Discussion}

The major finding of our study is that the peripheral smear review rate in outpatients was $7.2 \%$, and the availability of historical data decreased the rate to $2.6 \%$. Our lower smear rate allowed us to report all results within $8 \mathrm{~h}$ (turn around time), without an

Table 2 Proportion of 39,759 complete blood counts requiring reflex peripheral blood smear review.

\begin{tabular}{ll}
\hline According to the following criteria & Total $\mathrm{n}(\%)$ \\
\hline Basic 5 criteria $^{\mathrm{a}}$ & $1067(2.7)$ \\
+ Absolute lymphocytes $>5 \times 10^{9}$ cells/L & $1758(4.4)$ \\
+ Monocytes $>12 \%$ & $1940(4.9)$ \\
+ Neutrophils $<1 \times 10^{9}$ cells/L & $1966(4.9)$ \\
$+\mathrm{MCV} \geq 100 \mathrm{fL}$ & $2418(6.1)$ \\
$+\mathrm{LUC} \geq 5 \%$ & $2706(6.8)$ \\
+ Blast flag & $2746(6.9)$ \\
+ Immature granulocyte flag (three plus) & $2843(7.2)$ \\
With the availability of historical data & $1028(2.6)$ \\
\hline Add criteria of the consensus committee & \\
+ Immature granulocyte flag (one plus) & $4093(10.3)$ \\
+ Nucleated red cell flag (one plus) & $4405(11.1)$ \\
+ Eosinophils $>2000 \times 10^{9}$ cells $/ \mathrm{L}$, & $4459(11.2)$ \\
basophils $>0.5 \times 10^{9}$ cells $/ \mathrm{L}$ or RDW $>22 \%$ & \\
+ MCV $<75$ fL & $5988(15.1)$ \\
(Other minor corrections) ${ }^{\mathrm{b}}$ & $5741(13.9)$ \\
\hline
\end{tabular}

aWhite blood count $(\mathrm{WBC})<3.8 \times 10^{9}$ cells $/ \mathrm{L}, \mathrm{WBC} \geq 20 \times 10^{9}$ cells $/ \mathrm{L}$, platelets $<100$ or $>1000 \times 10^{9} / \mathrm{L}$, and hemoglobin $<70 \mathrm{~g} / \mathrm{L}$. ${ }^{b} \mathrm{WBC}<4 \times 10^{9}$ cells $/ \mathrm{L}, \mathrm{WBC}>30,000 \times 10^{9} \mathrm{cells} / \mathrm{L}$, monocytes $>1.500 \times 10^{9} \mathrm{cells} / \mathrm{L}$ and lymphocytes according to age as recommended by the consensus committee. 


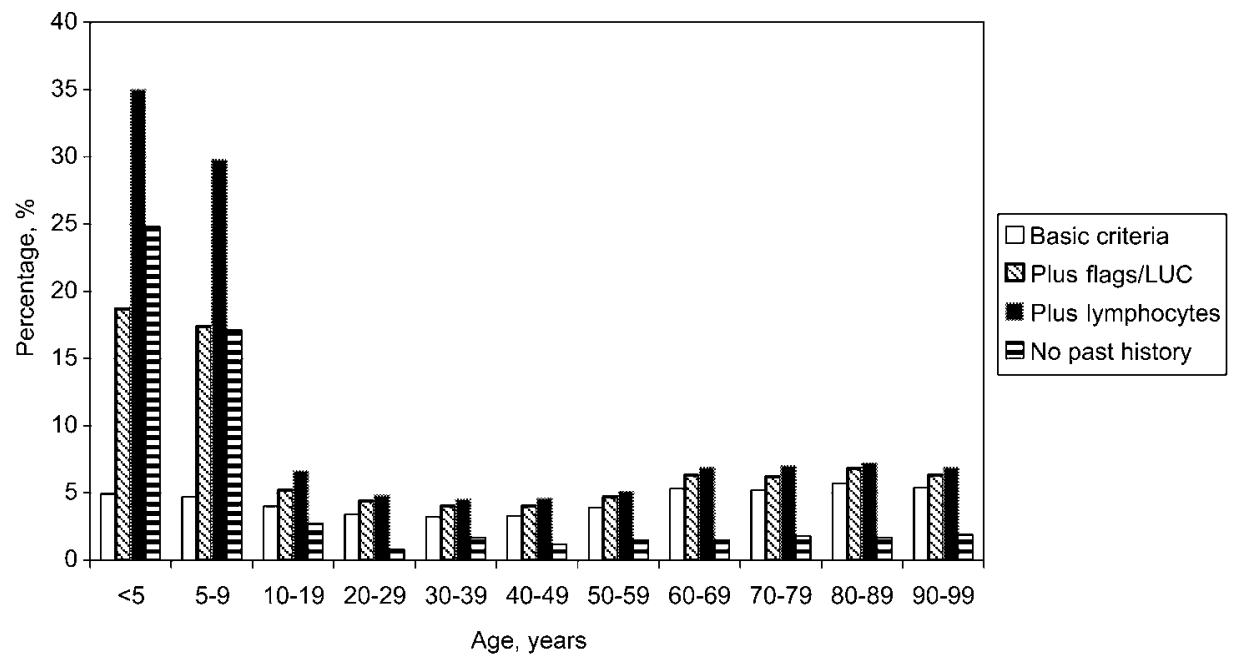

Figure 1 Indications and past history of peripheral blood smears.

The basic criteria are $\mathrm{Hb}<70 \mathrm{~g} / \mathrm{L}, \mathrm{WBC}>20$ or $<3.7 \times 10^{9} \mathrm{cells} / \mathrm{L}$, platelets $<100$ or $>999 \times 10^{9} / \mathrm{L}$, monocytes $>12 \%, \mathrm{MCV}>99$ $\mathrm{fL}$, or neutrophils $<1 \times 10^{9}$ cells/L. In those under age 10 years the basic criteria are similar to those 10 years and older, but flag indications/LUC (immature granulocytes +++ , blast flags, or large unstained lymphocytes $\geq 5 \%$ ) greatly increase the indications. A further increase is found when adding an absolute lymphocyte count of $>5 \times 10^{9}$ cells/L. Also, those without a previous smear done for the same indication are much more prevalent in those under 10 years of age.

observable loss in diagnostic efficiency. Although the proper cut-off value for the various criteria is unclear, it is likely that the availability of historical data using the consensus criteria will also result in a significant decrease in the need for peripheral smear reviews.

Our criteria were significantly different from those of a consensus committee (5), where trigger values in our population would have led to a smear review rate of $13.9 \%$. This is primarily due to the addition of smear reviews in those with an $\mathrm{MCV}<75 \mathrm{fL}$, and the use of a more sensitive cut-off for the immature granulocyte flag ( + instead of +++ ). Also, the lower cutoff value for atypical cells and a higher cut-off value for a decreased absolute WBC were contributing factors.

We did not perform slide reviews in patients with microcytosis. A recent study found that detailed slide reviews were not useful in differentiating between iron deficiency anemia, $\beta$-thalassemia minor and anemia of chronic disease (8). We perform reflex testing using hemoglobin electrophoresis if not previously done in samples with MCV values $<75 \mathrm{fL}$ and $\mathrm{RBCs}$ $\geq 4.5 \times 10^{12} / \mathrm{L}$. This led to a new diagnosis of $\beta$-thalassemia minor in 135 patients (67.8\% of those tested). Hemoglobin electrophoresis is performed in pregnant women according to ethnicity, regardless of their MCV.

Another consensus committee criterion that significantly increased the proportion of triggering values was a more conservative use of the immature granulocyte flag (any positive vs. our criteria of a plus three value). We did not find that the immature granulocyte flag independently identified patients with acute leukemia. Also, there is no evidence that automated CBC tests or manual peripheral blood smear reviews are useful in differentiating between viral and bacterial infectious diseases resulting in changes in patient treatment $(9,10)$.
We used lower cut-off values for the absolute WBC, and higher cut-off values for atypical lymphocytes compared with those suggested by the consensus committee. However, all patients with acute leukemia had both blast flags and elevated LUC counts or the basic criteria (anemia, thrombopenia, leukopenia or leukocytosis). We would have missed 5 of the 24 patients without the availability of the LUC counts and blast flags, but it is unclear whether other types of analyzers or even the Advia analyzers using a different calibration would have detected these five patients. Also, we cannot rule out whether there were other patients with acute leukemia who were missed because no peripheral smear was done. However, the large number of peripheral smears performed without detecting an additional case suggests that our criteria are appropriate for outpatients in our setting.

Using the consensus committee criteria, the results from the Advia analyzers are more specific for adults compared with children. In children, there are a very high proportion of tests with triggers due to LUC counts, flags for blasts and a three plus flag for immature granulocytes. We did not find a single case of acute leukemia in those under 10 years of age from over 20,000 smears that were reviewed during the entire year. The indications for blood counts in this age group are primarily routine screening testing at age 12 months, and for differentiating viral from bacterial infection. This supports the use of the agespecific criteria for children as was recommended for the absolute lymphocyte counts by the consensus committee. However, it is unclear if the blast flags and an increase in atypical lymphocytes found in over $15 \%$ of children can be ignored.

The strength of our study is that we have a welldefined population with utilization rates. It is likely that our results can be extrapolated to other similar settings. In hospitals however, illnesses of greater 
severity will lead to an increased number of triggering values for peripheral smears (4), and increased requests by physicians for peripheral smear review in specific cases might influence the diagnosis and treatment of hospitalized patients that are ill (3).

\section{Conflict of interest}

We have not accepted funding or support or employment by an organization that might gain financially from the results of this study. We have no conflict of interest.

\section{References}

1. Pierre RV. Peripheral blood film review: the demise of the eye-count leukocyte differential. Clin Lab Med 2002;22: 279-97.

2. Buttarello M, Gadotti M, Lorenz C, Toffalori E, Ceschini N, Valentini $A$, et al. Evaluation of four automated hematology analyzers: a comparative study of differential counts (imprecision and inaccuracy). Am J Clin Pathol 1992;97: 345-52.

3. Bain BJ. Diagnosis from the blood smear. N Engl J Med 2005;353:498-507.
4. Novis DA, Walsh M, Wilkinson D, St Louis M, Ben-Ezra J. Laboratory productivity and the rate of manual peripheral blood smear review. A College of American Pathologists Q-probes study of 95141 Complete blood count determinations performed in 263 Institutions. Arch Pathol Lab Med 2006;130:596-601.

5. International Consensus Group for Hematology Review, International Society for Laboratory Hematology. Suggested criteria for action following automated $\mathrm{CBC}$ and WBC differential analysis. Available at: http://www.islh. org/2004/Committees/ConsensusGroup/CGICGHREview. htm. Accessed March 17, 2009.

6. Froom P, Neck A, Shir M, Haavis R, Barak M. Automatic laboratory-initiated reflex testing to identify patients with autoimmune hemolytic anemia. Am J Clin Pathol 2005; 124:129-32.

7. Mustard CA, Kaufert P, Kozyrskyj A, Mayer T. Sex differences in the use of health care services. $N$ Engl J Med 1998;338:1678-83.

8. Harrington AM, Ward PC, Kroft SH. Iron deficiency anemia, beta-thalassemia minor and anemia of chronic disease. A morphologic reappraisal. Am J Clin Path 2008; 129:466-71.

9. Buttarello M, Plebani M. Automated blood cell counts. Am J Clin Pathol 2008;130:104-16.

10. Cornbleet PJ. Clinical utility of the band count. Clin Lab Med 2002;22:101-31. 